\title{
Collaboration in networks with randomly chosen agents*
}

\author{
Zhiwei Cui ${ }^{\dagger}$ Rui Wang ${ }^{\ddagger}$
}

\begin{abstract}
The present paper considers a finite population of agents located in an arbitrary, fixed network. In each period, a small proportion of agents are randomly chosen to play a minimum effort game. They learn from both their own and their neighbors' experiences and imitate the most successful choices, though they may occasionally make mistakes. We show that in the long run all agents will choose the highest effort level provided that each agent's neighborhood is large.
\end{abstract}

JEL: C72; D83

Keywords: Minimum effort game; Rare interaction; Local observation; Imitation

${ }^{*}$ We are indebted to Professor Dirk Engelmann, the associate editor and two anonymous referees for suggesting ways to improve both the content and exposition of this paper. We are also grateful to Zhigang Cao, Ge Jiang, Jonathan Newton, Fei Shi and Simon Weidenholzer for helpful comments and suggestions. This paper was presented at the 2014 Beijing Economic Theory Workshop at the University of International Business and Economics, International Conference on Multilateral Economic Cooperation Strategy under "One Belt One Road" Framework and Research Development on Game Theory and seminars at the Academy of Mathematics and Systems Science in the Chinese Academy of Sciences; Beijing Normal University; National Tsing Hua University; Renmin University of China; University of York; Shanghai Jiao Tong University and Zhejiang University. We greatly acknowledge all participants for their comments. This work was financially supported by the National Science Foundation of China (No.61202425) and the Fundamental Research Funds for the Central Universities (YWF-13-D2-JC-11, YWF-14-JGXY-016).

${ }^{\dagger}$ School of Economics and Management, Beihang University, Beijing 100191, PR China. Email: zhiweicuisx@gmail.com

${ }^{\ddagger}$ School of Computer Science and Engineering, Beihang University, Beijing 100191, PR China. Email: wangrui@buaa.edu.cn

(C) 2016. This manuscript version is made available under the Elsevier user license http://www.elsevier.com/open-access/userlicense/1.0/ 


\section{Introduction}

Interaction within networks is an active research field for economists (for an overview, see Goyal, 2011 and Özgür, 2011). The literature usually explores local interaction, where individuals only interact with their direct neighbors. Nevertheless, in a wide range of economic and social situations, individuals interact with strangers and interaction between the same group of individuals occurs with low frequency. Examples of this include peer-to-peer file sharing and online anonymous financial transaction services. It is quite common that each time only a small proportion of all agents take part in the interaction. In order to capture these phenomena, the present paper assumes that in each period, a fixed number of agents are randomly chosen from a finite population to interact and that these selected agents make their choices based on their own and their neighbors' experiences.

More specifically, let us concentrate on the example of peer-to-peer file sharing. Typically, a large file is broken down into much smaller chunks. These chunks can be obtained from multiple peers by each peer uploading the downloaded chunks to others. Each peer is able to determine his own uploading speed. The higher the uploading speed, the more bandwidth is occupied; then, the more the peer has to pay. Typically, how quickly file sharing occurs is determined by the slowest speed.

Hence, we consider a finite population of agents located in an arbitrary, fixed network. In each period, a fixed number of individuals from the whole population are randomly chosen to play a minimum effort game. Players choose among different effort levels and their payoffs depend on the minimum effort chosen by all players. Minimum effort games model situations where the performance of a group is determined by the lowest effort individual member. ${ }^{1}$ Each selected agent is assumed to observe the effort levels and payoffs of his neighbors and himself, in the most recent interaction and to imitate the most successful choice. Occasionally, agents make mistakes when revising their choices.

We find that if each agent has a large number of neighbors, in the long run all agents will choose the highest effort level and the socially optimal outcome will obtain provided that the probability of random noise is sufficiently small. ${ }^{2}$ In the language of economic and social networks, highly cohesive networks can lead to the socially optimal outcome. ${ }^{3}$ We also show that when one agent does not have enough neighbors, in the long run all agents will exert the lowest effort level and only the socially worst outcome occurs.

\footnotetext{
${ }^{1}$ The minimum effort games can be used to model collaboration among individuals (Alós-Ferrer and Weidenholzer, 2014 and Van Huyck, Battalio, and Beil, 1990).

${ }^{2}$ By conducting laboratory experiments with a minimum effort game, Weber (2006) shows that slow growth and exposure of entrants to previous norms can alleviate the problem of large group coordination failure.

${ }^{3}$ Given that social and economic networks are described precisely as undirected graphs, Seidman (1983) proposes that the minimum degree of all nodes can be used as a measure of network cohesion.
} 
The intuition underlying the main results is as follows. For simplicity, consider a connected observation network. If, in a given period, all selected agents exert the highest effort level, they will receive the maximal possible payoff. With positive probability, in the next period, their neighbors are selected and owing to the imitation rule will choose the highest effort level. Following this logic, the highest effort level can spread to the whole population. Now consider the reverse transition. First, assume that some player has strictly less neighbors than participants in the minimum effort game. If this agent and all of his neighbors are selected to play the minimum effort game and he makes a mistake and switches to the lowest effort level then this seed agent will earn the highest payoff. Following this interaction, assume that the seed agent is chosen again and interacts only with agents who are not neighbors. These new interaction partners will now earn a payoff that is no larger than the payoff of the seed agent. By consecutively selecting the appropriate interaction partners the payoffs of all agents can be lowered below the payoff of the seed agent. From this point onwards, the seed agent's neighbors will copy the lowest effort level with certainty. One can extend this argument to the case where the size of the neighborhood of the seed agent is larger than the number of participants in the minimum effort game. Doing so, one has to first make sure that none of the seed agent's neighbors earns a payoff higher than the seed agent (which would make him switch back immediately). Naturally, the larger the size of the smallest neighborhood the more mistakes will this take. For the transition mechanisms above, it is relative size rather than the identity of the highest or lowest effort level that matters. In most cases, there are only two candidates for stochastically stable equilibria: socially optimal and worst outcomes; the minimum degree decides which of these will emerge.

Our work contributes to research on local interaction. Starting with the seminal papers of Blume (1993, 1995) and Ellison (1993), much of the literature is concerned with local interaction games. It is assumed that agents are located in a fixed network and interact with their direct neighbors (Alós-Ferrer and Weidenholzer, 2007, 2008, 2014; Boyer and Jonard, 2014; Cui, 2014; Ellison, 2000; Eshel, Samuelson and Shaked, 1998 and Morris, 2000). Two prominent dynamic adjustment rules are used in these models: myopic best-response reply and imitation (for an overview, see Weidenholzer, 2010). As a complement, the present paper takes into account the possibility that individuals may interact with strangers and analyzes the long-run behavior of imitation dynamics.

Our paper also relates to research that explores the effect of random matching on learning dynamics in games. Building on Kandori, Mailath and Rob (1993), Robson and Vega-Redondo (1996) show that for a coordination game, a random pairing-up mechanism can lead to the emergence of the Pareto efficient rather than risk dominant equilibrium. And, given that agents are located in regular networks, Anderlini and Ianni (1996) introduce the concept of 1-factor which enables each agent to be coupled with one neighbor and show that in the long run different actions of a coordination game may survive at different locations. Although opponents may change over 
time, these papers assume that in each period, each individual plays a $2 \times 2$ coordination game with someone else. In contrast to these papers, the present paper assumes that in each period, only a small proportion of agents are selected to play a minimum effort game.

One closely related paper is that of Khan (2014) who proposes a model of global random interaction and local observation on a fixed network. Khan (2014) assumes that in each period all individuals are randomly partitioned into pairs to play a $2 \times 2$ coordination game and update their choices by imitating their most successful neighbors. Khan (2014) shows that the Pareto efficient convention is always stochastically stable and offers sufficient conditions for this outcome to be a unique stochastically stable equilibrium. Besides the difference in the base game, the present paper differs from Khan (2014) by assuming that in each period, only a small fraction of individuals interact rather than the whole population. Owing to rare and random interaction, the lowest effort level can spread from one agent and neighbors to the whole population even without any further mutation provided that the observation network is connected. Therefore, the socially optimal outcome may not be stochastically stable, even if the minimum effort game is reduced to a $2 \times 2$ coordination game (Proposition 2 in Section 4).

Another closely related paper is that of Alós-Ferrer and Weidenholzer (2014) where each individual plays a minimum effort game with all direct neighbors and an imitation rule is employed. They show that being able to observe agents who are not direct neighbors may help overcome the coordination problem. ${ }^{4}$ That is, besides interaction neighbors given by direct neighbors, each agent's information neighborhood contains strictly more elements. However, in the present paper, for each selected agent, information neighbors coincide with direct neighbors while interaction neighbors are randomly given. We find that the socially optimal outcome can be attained if, for each agent, the number of information neighbors is large enough. Although both papers require a large information neighborhood, different conditions on networks are imposed. To see this point, consider a complete network. In Alós-Ferrer and Weidenholzer (2014), for each agent, information neighborhood and interaction neighborhood coincide, and in the long run each agent exerts the lowest effort level. However, in our setting, which outcome turns out to be stochastically stable depends on the size of information neighborhood (Corollary 3 in Section 3.2.2).

The rest of the paper is organized as follows. Section 2 describes the basic building blocks of our model, including the minimum effort game, observation network and imitation dynamics. Section 3 investigates the convergence of unperturbed dynamics and stochastic stability of perturbed dynamics. Section 4 illustrates the main results using a stag-hunt game. Section 5 explores the robustness of the main findings with respect to a couple of changes in assumptions. Finally, Section 6 concludes.

\footnotetext{
${ }^{4}$ Angus and Masson (2010) assume that information and interaction are specified by two distinct but fixed networks and use simulation to show the effect of this distinction on contagion process.
} 


\section{The model}

\subsection{Minimum effort game}

The base game is an $n$-person minimum effort game $\Gamma$ with $n \geq 2$. In the minimum effort game, each player's payoff depends on the minimum among all players' effort levels.

Let $\mathbf{N} \doteq\{1, \cdots, n\}$ be the set of players. Each player $i$ chooses a level of effort, $e_{i}$, from the common set $E \doteq\left\{e^{1}, \cdots, e^{m}\right\}$ where $m>1$ and $0<e^{1}<\cdots<e^{m}$. A strategy profile is represented as $\left(e_{1}, \cdots, e_{n}\right)$. Let $\vec{e}$ denote the strategy profile where all players choose the same effort level $e$ for any $e \in E$.

For each choice $e_{i}$, player $i$ bears a cost $\delta e_{i}$ where $0<\delta<1$. For any strategy profile $\left(e_{1}, \cdots, e_{n}\right)$, player $i$ 's payoff is

$$
\pi_{i}\left(e_{1}, \cdots, e_{n}\right)=\min _{1 \leq j \leq n} e_{j}-\delta e_{i}
$$

For each player, the maximal payoff brought about by effort level $e$ is $(1-\delta) e$ which can be attained when other players' choices are no less than $e$. Thus, the maximal possible payoff is $(1-\delta) e^{m}$. The set of Nash equilibria is $\{\vec{e}: e \in E\}$ where each $\vec{e}$ is a strict Nash equilibrium.

\subsection{Network and local observation}

Let $\mathbf{I} \doteq\{1, \cdots, I\}$ be the set of agents with $I \geq n$. For any two agents $i, j \in \mathbf{I}$, the pairwise relationship between them is captured by a binary variable, $g_{i j} \in\{0,1\}$. We write $g_{i j}=1$ if agent $i$ can observe agent $j$ 's effort level and payoff and $g_{i j}=0$ otherwise; conventionally, $g_{i i}=1$. The relationship is assumed to be symmetric; formally, $g_{i j}=g_{j i}$. In other words, there is a dichotomy: mutual observation and no observation. We say $g \doteq\left\{\left(g_{i j}\right)_{i, j \in \mathbf{I}}\right\}$ defines an observation network over the set $\mathbf{I}$, and the undirected graph $\mathbf{g} \doteq(\mathbf{I}, g)$ defines an observation system.

For any agent $i \in \mathbf{I}$, the information neighborhood $M(i) \doteq\left\{j \in \mathbf{I}: g_{i j}=1\right\}$ is the set of agents whose effort levels and payoffs can be observed. Assume that $M(i) \backslash\{i\} \neq \emptyset$; that is, except himself, agent $i$ can observe at least one another agent. Each element of $M(i) \backslash\{i\}$ will be referred to as a neighbor of agent $i$.

For any subset $\mathbf{I}^{\prime} \subseteq \mathbf{I}$, the subgraph $\left(\mathbf{I}^{\prime},\left(g_{i j}\right)_{i, j \in \mathbf{I}^{\prime}}\right)$ is a component of $\mathbf{g}$ if $(1)$ for any $i$ and $j$ from $\mathbf{I}^{\prime}$, there exists $\left\{i_{0}, \cdots, i_{L-1}\right\} \cup\left\{i_{L}\right\} \subseteq \mathbf{I}^{\prime}$ such that $i_{0}=i, i_{L}=j$ and $g_{i_{l} i_{l+1}}=1$ for any $l=0, \cdots,(L-1)$ and (2) there is no strict superset $\mathbf{I}^{\prime \prime}$ of $\mathbf{I}^{\prime}$ such that (1) holds. Let $\mathbf{c}=\left(\mathbf{I}^{\prime},\left(g_{i j}\right)_{i, j \in \mathbf{I}^{\prime}}\right)$ denote a component, and $\mathbf{I}^{\prime}$ will be referred to as the set of members. For simplicity, we use $i \in \mathbf{c}(i \notin \mathbf{c})$ to represent $i \in \mathbf{I}^{\prime}\left(i \notin \mathbf{I}^{\prime}\right)$. Let $|\mathbf{c}|$ be the number of agents in $\mathbf{c}$. Let $\eta(g)$ be the number of components of $\mathbf{g}$. We say $g$ is connected if $\mathbf{g}$ has a unique component. 


\subsection{Random sampling and imitation dynamics}

Let $\mathcal{I}^{n} \doteq\left\{\mathbf{I}^{\prime}: \mathbf{I}^{\prime} \subseteq \mathbf{I}\right.$ and $\left.\left|\mathbf{I}^{\prime}\right|=n\right\}$ denote the set of all possible subsets of $\mathbf{I}$ with cardinality $n .{ }^{5}$ Assume that the random sampling mechanism $\nu$ is a probability distribution over $\mathcal{I}^{n}$, satisfying that $\nu\left(\mathbf{I}^{\prime}\right)>0$ for any $\mathbf{I}^{\prime} \in \mathcal{I}^{n}$.

Time is discrete; $t=0,1,2, \ldots$. In each period $t$, a group $\mathbf{I}^{\prime}(t) \in \mathcal{I}^{n}$ of $n$ distinct agents is randomly chosen with probability $\nu\left(\mathbf{I}^{\prime}(t)\right)>0$ to play the minimum effort game $\Gamma$. The number of selected agents, $n$, is assumed to stay fixed as time moves on. ${ }^{6}$ Let $X \doteq\left\{\left(e, e^{\prime}-\delta e\right): \forall e, e^{\prime} \in\right.$ $E$ and $\left.e \geq e^{\prime}\right\}$ be the set of all possible states for any agent $i \in \mathbf{I}$.

Let $s_{i}(t)=\left(e_{i}(t), u_{i}(t)\right) \in X$ be agent $i$ 's state which specifies his effort level and payoff in the most recent interaction until period $t+1$. The state $s_{i}(t)$ is updated as follows. First, consider the case that $i \in \mathbf{I}^{\prime}(t)$. If $t=0$, agent $i$ randomly chooses $e_{i}(t)$ from $E$; otherwise, agent $i$ observes the effort levels and payoffs of his neighbors and himself in the most recent interaction, and imitates the most successful choice. ${ }^{7}$ Formally,

$$
e_{i}(t) \in\left\{e_{j}(t-1): j \in M(i) \text { and } u_{j}(t-1) \geq u_{j^{\prime}}(t-1) \text { for } \forall j^{\prime} \in M(i)\right\} .
$$

If the set given by the right-hand side of Equation (1) is not a singleton, every element will be chosen with strictly positive probability. Then, all members of $\mathbf{I}^{\prime}(t)$ play the game $\Gamma$ with $\left\{e_{j}(t)\right\}_{j \in \mathbf{I}^{\prime}(t)}$. Agent $i$ receives a payoff

$$
u_{i}(t)=\min _{j \in \mathbf{I}^{\prime}(t)} e_{j}(t)-\delta e_{i}(t)
$$

In addition, for any agent $i \notin \mathbf{I}^{\prime}(t)$, if $t=0, s_{i}(t)$ is randomly chosen from $X$ where each element will be chosen with strictly positive probability and if $t \geq 1, s_{i}(t)=s_{i}(t-1)$.

Let $\Omega \subset X^{I}$ be the set of all possible states specifying all agents' effort levels and payoffs in the most recent interaction; formally, a state $s=\left(\left(e_{1}, u_{1}\right), \cdots,\left(e_{I}, u_{I}\right)\right) \in \Omega$ if and only if $s \in X^{I}$ and there exists $i_{0} \in \mathbf{I}$ such that

$$
u_{i_{0}}=e_{i_{0}}-\delta e_{i_{0}} \text { and }\left|\left\{i \in \mathbf{I}: u_{i}=e_{i_{0}}-\delta e_{i}\right\}\right| \geq n .
$$

The existence of agent $i_{0}$ says that a group of $n$ agents, including $i_{0}$, is selected to play the game $\Gamma$ where the minimum of all players' choices is $e_{i_{0}}$. For any $e \in E$, we use $\overrightarrow{(e, e-\delta e)}$ to denote the state $((e, e-\delta e), \cdots,(e, e-\delta e)) \in \Omega$.

\footnotetext{
${ }^{5}$ For any finite set $X$, the cardinality $|X|$ is the number of elements belonging to $X$.

${ }^{6}$ In section 5 , we show that the main results are robust to variation in the number of selected agents.

${ }^{7}$ This feature relates the present paper with the literature on imitation dynamics where memory is explicitly introduced (Alós-Ferrer, 2004, 2008 and Bergin and Bernhardt, 2009). Our model has no requirement on the length of memory. It assumes that agents have a memory of the most recent experiences of their neighbors, as well as themselves.
} 
The above strategy revision process defines a Markov chain $\{S(t)\}_{t \in \mathbb{N}}$ over the state space $\Omega$. An absorbing set is a minimal subset of $\Omega$ with the property that, under the unperturbed dynamics, the probability of exit from it is zero. So the possible states the unperturbed dynamics will converge to are states contained in absorbing sets. An absorbing state is an element which forms a singleton absorbing set. For any absorbing set $\bar{\Omega}$, the basin of attraction $D(\bar{\Omega})$ is the set of states from which the unperturbed dynamics will converge to $\bar{\Omega}$ with probability one.

There exists a multiplicity of absorbing sets. For instance, for any $e \in E, \overrightarrow{(e, e-\delta e)}$ is an absorbing state. When agent $i$ is randomly chosen to play the game $\Gamma, i$ observes that all members of $M(i)$ exert the same effort level $e$ and get the same payoff $(1-\delta) e$. Then, agent $i$ will choose $e$ and receive a payoff $(1-\delta) e$.

To select among all possible absorbing sets, we introduce random noise (Kandori, Mailath and Rob, 1993 and Young, 1993). Suppose that, when selected to participate in the game $\Gamma$, each agent $i$ makes a mistake with probability $\epsilon, \epsilon \in(0,1)$. In this case, $i$ randomly chooses his effort level. The most common specification for mistakes in the economics literature is for random noise to be independent across agents and periods. Therefore, the perturbed dynamics $\left(\left\{S^{\epsilon}(t)\right\}_{t \in \mathbb{N}}, \Omega\right)$ are defined. For any $\epsilon \in(0,1),\left\{S^{\epsilon}(t)\right\}_{t \in \mathbb{N}}$ is an irreducible and aperiodic Markov chain and has a unique invariant distribution $\mu^{\epsilon}$. A state $s$ is stochastically stable if $\mu^{*}(s)>0$ where $\mu^{*}=\lim _{\epsilon \rightarrow 0} \mu^{\epsilon}$.

\section{Main results}

Given the building blocks of the model, this section examines the likelihood of the emergence of the highest effort convention. We aim to identify sufficient condition under which, in the long run, every selected agent will choose the highest effort level. With this goal in mind, we investigate the convergence of the unperturbed dynamics and stochastic stability of the perturbed dynamics.

\subsection{Convergence of unperturbed dynamics}

In the following proposition, we characterize the absorbing sets of the unperturbed dynamics. The proof is provided in the Appendix.

Proposition 1. Consider the unperturbed dynamics $\{S(t)\}_{t \in \mathbb{N}}$. For any two states

$$
s=\left(\left(e_{1}, u_{1}\right), \cdots,\left(e_{I}, u_{I}\right)\right) \text { and } s^{\prime}=\left(\left(e_{1}^{\prime}, u_{1}^{\prime}\right), \cdots,\left(e_{I}^{\prime}, u_{I}^{\prime}\right)\right)
$$

in an absorbing set $\bar{\Omega}, e_{i}=e_{j}=e_{i}^{\prime}=e_{j}^{\prime}$ for every two members $i$ and $j$ of each component of $\mathbf{g}$.

Proposition 1 shows that for each absorbing set all members of any component of $\mathrm{g}$ exert the same effort level. Consider a component $\mathbf{c}$ where $|\mathbf{c}| \leq n$. Assume that in periods $t_{0}$ and 
$t_{0}+1$, all agents in $\mathbf{c}$ are selected to play the game $\Gamma$. Suppose that the deterministic imitation rule requires that agent $i_{0} \in \mathbf{c}$ chooses effort level $e^{1}$ in period $t_{0}$. Then, in period $t_{0}$, each agent $j \in \cup_{k \in M\left(i_{0}\right)} M(k)$ performs worse than $i_{0}$. All agents in $M\left(i_{0}\right)$ exert $e^{1}$ in period $t_{0}+1$. Following this logic, $e^{1}$ can spread from $i_{0}$ to $i_{0}$ 's neighbors, and finally to all members of $\mathbf{c}$.

For a disconnected observation network $g$, all absorbing sets can be divided into two types. When all individuals in I choose the same effort level $e$, we have a monomorphic absorbing state $\overrightarrow{(e, e-\delta e)}$. However, when there exist two distinct components such that their members choose different effort levels, the absorbing set is not a singleton.

Insert Figure 1 here.

To see this point, consider a society $\mathbf{I}=\{1, \cdots, 7\}$ where individuals are randomly chosen to play a 2-person minimum effort game. The observation system g consists of two components:

$$
\begin{aligned}
& \mathbf{c}_{1}=\left(\{1,2,3\}, g_{12}=g_{23}=g_{13}=1\right), \\
& \mathbf{c}_{2}=\left(\{4,5,6,7\}, g_{45}=g_{56}=g_{67}=g_{74}=1\right) .
\end{aligned}
$$

As an illustration, see Figure 1. Suppose that in some period $t_{0}$, all agents from $\mathbf{c}_{1}\left(\mathbf{c}_{2}\right)$ exert the same effort level $e^{1}\left(e^{2}\right)$ in the most recent interaction. Following the imitation rule specified by Equation (1), all agents from $\mathbf{c}_{1}\left(\mathbf{c}_{2}\right)$ will choose $e^{1}\left(e^{2}\right)$ forever. However, when selected to play the game $\Gamma$, agent $i$ 's, $i \in \mathbf{c}_{1}$, payoff will depend on whether or not the other player is from $\mathbf{c}_{1}$ and cannot be a constant amount. Therefore, $\{S(t)\}_{t \in \mathbb{N}}$ may converge to an absorbing set consisting of multiple states.

As an immediate result, Proposition 1 yields the following corollary for connected observation networks.

Corollary 1. Assume that the observation network $g$ is connected. Then the unperturbed dynamics $\{S(t)\}_{t \in \mathbb{N}}$ will converge to one of the absorbing states.

\subsection{Stochastic stability of perturbed dynamics}

In this section, we first explore stochastic stability of the perturbed dynamics for general observation networks. Then, we restrict our attention to connected observation networks.

\subsubsection{General observation networks}

Before proceeding to the result concerning the long-run behavior of the perturbed dynamics, we provide three lemmas that characterize the traverse across different absorbing sets. The proofs of 
these lemmas and the main theorem are provided in the Appendix.

Consider an absorbing set $\bar{\Omega}$. Let $e^{h}(\bar{\Omega})$ and $e^{l}(\bar{\Omega})$ be the highest and lowest effort levels chosen by agents in $\bar{\Omega}$. By Proposition $1, e^{h}(\bar{\Omega})$ and $e^{l}(\bar{\Omega})$ are independent of which state in $\bar{\Omega}$ is realized. For any $\left(\left(e_{1}, u_{1}\right), \cdots,\left(e_{I}, u_{I}\right)\right) \in \bar{\Omega}$ and $i \in \mathbf{I}, e^{l}(\bar{\Omega}) \leq e_{i} \leq e^{h}(\bar{\Omega})$ and there exist components c and $\mathbf{c}^{\prime}$ such that $e_{i}=e^{l}(\bar{\Omega})$ for all $i \in \mathbf{c}$ and $e_{j}=e^{h}(\bar{\Omega})$ for all $j \in \mathbf{c}^{\prime}$. Let $\rho(\bar{\Omega})$ be the number of agents exerting $e^{h}(\bar{\Omega})$.

The following lemma examines the transition from the non-monomorphic absorbing set $\bar{\Omega}$ to the absorbing state $\overrightarrow{\left(e^{l}(\bar{\Omega}), e^{l}(\bar{\Omega})-\delta e^{l}(\bar{\Omega})\right)}$.

Lemma 1. Consider the perturbed dynamics $\left\{S^{\epsilon}(t)\right\}_{t \in \mathbb{N}}, \epsilon \in(0,1)$. For any absorbing set $\bar{\Omega}$, $e^{h}(\bar{\Omega})>e^{l}(\bar{\Omega})$, there is a sequence of distinct absorbing sets $\left(\bar{\Omega}_{0}, \cdots, \bar{\Omega}_{k}\right)$ such that $(1) \bar{\Omega}_{0}=\bar{\Omega}$ and $\bar{\Omega}_{k}=\left\{\overrightarrow{\left(e^{l}(\bar{\Omega}), e^{l}(\bar{\Omega})-\delta e^{l}(\bar{\Omega})\right)}\right\} ;(2)$ for any $0 \leq k^{\prime} \leq(k-1), \bar{\Omega}_{k^{\prime}+1}$ is such that there is one more component with all members choosing $e^{l}(\bar{\Omega})$ than in $\bar{\Omega}_{k^{\prime}}$; and $(3)$ one single mutation can induce a transition from $\bar{\Omega}_{k^{\prime}}$ to $\bar{\Omega}_{k^{\prime}+1}$ for any $0 \leq k^{\prime} \leq(k-1)$.

Without loss of generality, assume that $e^{l}(\bar{\Omega})=e^{1}$. Consider a component $\mathbf{c}$ where $e_{i}=e>e^{1}$ for any $i \in \mathbf{c}$ and $s \in \bar{\Omega}$. When an agent $j_{0}$ who exerts $e^{1}$ and all members of $\mathbf{c}$ are consecutively selected to play the game $\Gamma$, if (1) $j_{0}$ is selected each time and chooses $e^{1}$ by following the unperturbed imitation rule and (2) some agent $i_{0} \in \mathbf{c}$ chooses $e^{1}$ by mistake, each agent $i \in \mathbf{c}$ obtains a lower payoff than $i_{0}$. Effort level $e^{1}$ then can spread from agent $i_{0}$ to all members of $\mathbf{c}$.

The following lemma explores the transition from an absorbing state to another absorbing state where all agents exert a strictly higher effort level and describes how it is possible to leave $D\left(\left\{\overrightarrow{\left(e^{1}, e^{1}-\delta e^{1}\right)}\right\}\right)$.

Lemma 2. Consider the perturbed dynamics $\left\{S^{\epsilon}(t)\right\}_{t \in \mathbb{N}}, \epsilon \in(0,1)$. For any $e, e^{\prime} \in E$ and $e<e^{\prime}$, there exists a sequence of distinct absorbing sets $\left(\bar{\Omega}_{0}, \cdots, \bar{\Omega}_{k}\right)$ such that

- $\bar{\Omega}_{0}=\{\overrightarrow{(e, e-\delta e)}\}$ and $\left.\bar{\Omega}_{k}=\left\{\overrightarrow{\left(e^{\prime}, e^{\prime}-\delta e^{\prime}\right.}\right)\right\}$;

- $e^{h}\left(\bar{\Omega}_{1}\right)=e^{\prime}, \rho\left(\bar{\Omega}_{1}\right) \geq n$ and $\bar{\Omega}_{k^{\prime}+1}$ is such that there is one more component with all members choosing $e^{\prime}$ than in $\bar{\Omega}_{k^{\prime}}$ for any $1 \leq k^{\prime} \leq(k-1)$; and

- to transit from $\bar{\Omega}_{0}$ to $\bar{\Omega}_{1}, n$ mistakes are required and sufficient and one single mutation can induce a transition from $\bar{\Omega}_{k^{\prime}}$ to $\bar{\Omega}_{k^{\prime}+1}$ for any $1 \leq k^{\prime} \leq(k-1)$.

In addition, to leave $D\left(\left\{\overrightarrow{\left(e^{1}, e^{1}-\delta e^{1}\right)}\right\}\right), \min \left\{n, \min _{\mathbf{c} \subseteq \mathbf{g}}|\mathbf{c}|\right\}$ mutations are needed and sufficient.

To make effort level $e^{\prime}$ earn a higher payoff than $e, n$ agents are required to choose $e^{\prime}$ and participate in the game $\Gamma$ simultaneously. These agents receive a payoff $(1-\delta) e^{\prime}$. Then, $e^{\prime}$ can spread to all agents who are connected to these $n$ agents in $g$. This explains the transition from $\bar{\Omega}_{0}$ to $\bar{\Omega}_{1}$. Now consider the transition from $\bar{\Omega}_{k^{\prime}}$ to $\bar{\Omega}_{k^{\prime}+1}$ for any $1 \leq k^{\prime} \leq(k-1)$. Assume that for 
component c, all members exert effort level $e$ in $\bar{\Omega}_{k^{\prime}}$. When $(n-1)$ agents who choose $e^{\prime}$ and agent $i_{0} \in \mathbf{c}$ are selected to play $\Gamma$, let agent $i_{0}$ mutate to play $e^{\prime}$. Agent $i_{0}$ gets a payoff $(1-\delta) e^{\prime}$ and performs better than any other member of $\mathbf{c}$. Then, $e^{\prime}$ can spread from agent $i_{0}$ to all members of $\mathbf{c}$. The transition from $\bar{\Omega}_{k^{\prime}}$ to $\bar{\Omega}_{k^{\prime}+1}$ is completed. In addition, when there is a component $\mathbf{c}$ such that $|\mathbf{c}| \leq n$, all members of $\mathbf{c}$ can be selected in one single period, and due to $|\mathbf{c}|$ mutations, they can switch from $e^{1}$ to $e \in E \backslash\left\{e^{1}\right\}$ simultaneously. This induces an exit from $D\left(\left\{\overrightarrow{\left(e^{1}, e^{1}-\delta e^{1}\right)}\right\}\right)$.

The following lemma analyzes the transit from an absorbing state to another absorbing state where all agents exert a strictly lower effort level and demonstrates how it is possible to leave $D\left(\left\{\overrightarrow{\left(e^{m}, e^{m}-\delta e^{m}\right)}\right\}\right)$.

Lemma 3. Consider the perturbed dynamics $\left\{S^{\epsilon}(t)\right\}_{t \in \mathbb{N}}, \epsilon \in(0,1)$. For any $e, e^{\prime} \in E$ and $e<e^{\prime}$, there exists a sequence of distinct absorbing sets $\left(\bar{\Omega}_{0}, \cdots, \bar{\Omega}_{\eta(g)}\right)$ such that

- $\left.\bar{\Omega}_{0}=\left\{\overrightarrow{\left(e^{\prime}, e^{\prime}-\delta e^{\prime}\right.}\right)\right\}$ and $\bar{\Omega}_{\eta(g)}=\{\overrightarrow{(e, e-\delta e)}\}$;

- $\bar{\Omega}_{k^{\prime}+1}$ is such that there is one more component with all members choosing e than in $\bar{\Omega}_{k^{\prime}}$ for any $0 \leq k^{\prime} \leq \eta(g)-1$; and

- to transit from $\bar{\Omega}_{0}$ to $\bar{\Omega}_{1}, \min _{i \in \mathbf{I}}\left\lceil\frac{|M(i)|}{n}\right\rceil$ mistakes are needed and sufficient; and one single mutation can induce a transition from $\bar{\Omega}_{k^{\prime}}$ to $\bar{\Omega}_{k^{\prime}+1}$ for any $1 \leq k^{\prime} \leq \eta(g)-1 .^{8}$

Thus, to leave $\left.D\left(\left\{\overline{\left(e^{m}, e^{m}-\delta e^{m}\right.}\right)\right\}\right), \min _{i \in \mathbf{I}}\left\lceil\frac{|M(i)|}{n}\right\rceil$ mutations are required and sufficient.

The transition from $\bar{\Omega}_{1}$ to $\bar{\Omega}_{\eta(g)}$ can be completed by applying Lemma 1 . It is sufficient to study the transition from $\bar{\Omega}_{0}$ to $\bar{\Omega}_{1}$. Without loss of generality, let us discuss how to leave $D\left(\left\{\overrightarrow{\left(e^{m}, e^{m}-\delta e^{m}\right)}\right\}\right)$. If there exists one agent $j \in M(i)$ whose state is $\left(e^{m},(1-\delta) e^{m}\right)$, then following the deterministic imitation rule, the selected agent $i$ chooses $e^{m}$. Equivalently, only if there exists an agent $i_{0}$ such that agent $j$ 's state is not given by $\left(e^{m},(1-\delta) e^{m}\right)$ for any $j \in M\left(i_{0}\right)$, then leaving $D\left(\left\{\overrightarrow{\left(e^{m}, e^{m}-\delta e^{m}\right)}\right\}\right)$ is possible. Now consider agent $i_{0} \in \arg \min _{i \in \mathbf{I}}|M(i)|$. If agent $i_{0}$ and his neighbors are consecutively selected to play the game $\Gamma$ and each time exactly one agent in $M\left(i_{0}\right)$ exerts effort level $e^{1}$ by mistake, then agent $j$ 's state is either $\left(e^{1},(1-\delta) e^{1}\right)$ or $\left(e^{m}, e^{1}-\delta e^{m}\right)$ for any $j \in M\left(i_{0}\right)$. Agent $i_{0}$ will play $e^{1}$ when selected in the next period. Then, $\left\{S^{\epsilon}(t)\right\}_{t \in \mathbb{N}}$ can converge to an absorbing set where all members of the component containing $i_{0}$ choose $e^{1}$. Owing to the fact that each time the game $\Gamma$ is played by $n$ selected agents among which one makes a mistake, $\left\lceil\frac{\left|M\left(i_{0}\right)\right|}{n}\right\rceil$ rather than $\left|M\left(i_{0}\right)\right|$ mutations are required and sufficient.

With the help of Lemmas 1-3, we are able to present our main result.

Theorem 1. Consider the perturbed dynamics $\left\{S^{\epsilon}(t)\right\}_{t \in \mathbb{N}}, \epsilon \in(0,1)$. Then,

\footnotetext{
${ }^{8}$ For any real number $x>0,\lceil x\rceil$ is the least integer no less than $x$.
} 
- if $\min _{i \in \mathbf{I}}\left\lceil\frac{|M(i)|}{n}\right\rceil>n$, only $\overrightarrow{\left(e^{m}, e^{m}-\delta e^{m}\right)}$ is stochastically stable;

- if $\min _{i \in \mathbf{I}}\left\lceil\frac{|M(i)|}{n}\right\rceil=n$, the set of stochastically stable equilibria is $\{\overrightarrow{(e, e-\delta e)}: e \in E\}$; and

- if $\min _{i \in \mathbf{I}}\left\lceil\frac{|M(i)|}{n}\right\rceil<n, \overrightarrow{\left(e^{1}, e^{1}-\delta e^{1}\right)}$ is a unique stochastically stable equilibrium.

Consider the case that agent $i_{0} \in \arg \min _{i \in \mathbf{I}}|M(i)|$ does not have enough neighbors; that is, $\left\lceil\frac{\left|M\left(i_{0}\right)\right|}{n}\right\rceil<n$. To leave $D\left(\left\{\overline{\left(e^{1}, e^{1}-\delta e^{1}\right)}\right\}\right), n$ mutations are required. And, when the initial state is $\overrightarrow{\left(e^{m}, e^{m}-\delta e^{m}\right)}$, it is possible that agent $i_{0}$ and his neighbors are consecutively selected to play the game $\Gamma$ with one player in $M\left(i_{0}\right)$ choosing $e^{1}$ each time by mistake. Effort level $e^{1}$ then is the most "successful" choice in $M\left(i_{0}\right)$, and can spread to all individuals who are from the same component as $i_{0}$. And, $\left\lceil\frac{\left|M\left(i_{0}\right)\right|}{n}\right\rceil$ mutations are required and sufficient. The basin of attraction $D\left(\left\{\overrightarrow{\left(e^{1}, e^{1}-\delta e^{1}\right)}\right\}\right)$ is therefore more difficult to exit than to enter it.

As an immediate result, Theorem 1 yields the following corollary.

Corollary 2. Consider the perturbed dynamics $\left\{S^{\epsilon}(t)\right\}_{t \in \mathbb{N}}, \epsilon \in(0,1)$. If $I \leq(n-1) n$, only $\overrightarrow{\left(e^{1}, e^{1}-\delta e^{1}\right)}$ is stochastically stable.

Given a small population, it is very easy for effort level $e^{1}$ to be the "most successful" choice in any information neighborhood and then spread to the entire population. Corollary 2 shows that if in each period, a relatively high proportion of agents are randomly chosen to play the game $\Gamma$, in the long run only $\overrightarrow{\left(e^{1}, e^{1}-\delta e^{1}\right)}$ will be observed with strictly positive probability provided that stochastic mutations vanish. It means that society is trapped into the worst equilibrium.

\subsubsection{Connected observation networks}

Corollary 1 illustrates that for connected observation networks, the unperturbed dynamics will converge to absorbing states. As a complement, by applying Theorem 1, we can provide the following characterization.

Corollary 3. Assume that the observation network $g$ is connected. Consider the perturbed dynam$\operatorname{ics}\left\{S^{\epsilon}(t)\right\}_{t \in \mathbb{N}}, \epsilon \in(0,1)$. Then,

- if $\min _{i \in \mathbf{I}}\left\lceil\frac{|M(i)|}{n}\right\rceil>n, \overline{\left(e^{m}, e^{m}-\delta e^{m}\right)}$ is a unique stochastically stable equilibrium;

- if $\min _{i \in \mathbf{I}}\left\lceil\frac{|M(i)|}{n}\right\rceil=n$, all absorbing states are stochastically stable; and

- if $\min _{i \in \mathbf{I}}\left\lceil\frac{|M(i)|}{n}\right\rceil<n$, only $\overrightarrow{\left(e^{1}, e^{1}-\delta e^{1}\right)}$ is stochastically stable. 


\section{Stag-hunt dilemma in networks with randomly chosen agents}

In this section, we consider a special case of minimum effort games where $n=2$ and $E$ consists of only $e^{1}$ and $e^{2}$. This $2 \times 2$ version of the game $\Gamma$ can be represented by the following table

\begin{tabular}{l|c|c|}
\multicolumn{2}{c}{$e^{1}$} & $e^{2}$ \\
\cline { 2 - 3 }$e^{1}$ & $(1-\delta) e^{1},(1-\delta) e^{1}$ & $(1-\delta) e^{1}, e^{1}-\delta e^{2}$ \\
\cline { 2 - 3 }$e^{2}$ & $e^{1}-\delta e^{2},(1-\delta) e^{1}$ & $(1-\delta) e^{2},(1-\delta) e^{2}$ \\
\cline { 2 - 3 } & &
\end{tabular}

where as before, $0<e^{1}<e^{2}$ and $0<\delta<1$. Let $\Gamma_{2,2}$ denote this $2 \times 2$ example of the game $\Gamma$.

By simple calculation, $\Gamma_{2,2}$ can be transformed into

\begin{tabular}{l|l|l|}
\multicolumn{2}{c}{} & \multicolumn{2}{c}{$e^{1}$} & \multicolumn{1}{c}{$e^{2}$} \\
\cline { 3 - 3 }$e^{1}$ & $\delta, \delta$ & $\delta, 0$ \\
\cline { 2 - 3 }$e^{2}$ & $0, \delta$ & 1,1 \\
\cline { 2 - 3 } & &
\end{tabular}

Note that $\Gamma_{2,2}$ is a stag-hunt dilemma where effort levels $e^{1}$ and $e^{2}$ correspond to strategies "Hare" and "Stag", respectively. $\Gamma_{2,2}$ has two strict Nash equilibria $\left(e^{1}, e^{1}\right)$ and $\left(e^{2}, e^{2}\right)$.

Proposition 1 shows that when the observation network $g$ is connected, there are only two possible absorbing sets $\left\{\overrightarrow{\left(e^{1}, e^{1}-\delta e^{1}\right)}\right\}$ and $\left\{\overrightarrow{\left(e^{2}, e^{2}-\delta e^{2}\right)}\right\}$. When $g$ is disconnected, there also exist other absorbing sets where agents from different components may exert different effort levels.

Theorem 1 then immediately yields the following proposition about the long-run behavior of the perturbed dynamics given that the game $\Gamma_{2,2}$ is repeatedly played by randomly chosen agents.

Proposition 2. Consider the perturbed dynamics $\left\{S^{\epsilon}(t)\right\}_{t \in \mathbb{N}}, \epsilon \in(0,1)$, for the stag-hunt game $\Gamma_{2,2}$. Then,

- if $\min _{i \in \mathbf{I}}\left\lceil\frac{|M(i)|}{2}\right\rceil>2$, only $\overrightarrow{\left(e^{2}, e^{2}-\delta e^{2}\right)}$ is stochastically stable ;

- if $\min _{i \in \mathbf{I}}\left\lceil\frac{|M(i)|}{2}\right\rceil=2$, stochastically stable equilibria are given by $\overrightarrow{\left(e^{1}, e^{1}-\delta e^{1}\right)}$ and $\overrightarrow{\left(e^{2}, e^{2}-\delta e^{2}\right)}$; and

- if $\min _{i \in \mathbf{I}}\left\lceil\frac{|M(i)|}{2}\right\rceil<2$, only $\overrightarrow{\left(e^{1}, e^{1}-\delta e^{1}\right)}$ is stochastically stable.

Assume that the observation network is connected. To induce a transition from $\overrightarrow{\left(e^{1}, e^{1}-\delta e^{1}\right)}$ to $\overrightarrow{\left(e^{2}, e^{2}-\delta e^{2}\right)}$, two mutations are needed and sufficient. When each agent has at least four neighbors, then exiting from $D\left(\left\{\overrightarrow{\left(e^{2}, e^{2}-\delta e^{2}\right)}\right\}\right)$ is more difficult than entering it. And, two mutations are sufficient and necessary to induce a transition from one absorbing state to the other when each agent has two or more neighbors and one agent has two or three neighbors. Finally, one single mutation can lead to a transition from $\overrightarrow{\left(e^{2}, e^{2}-\delta e^{2}\right)}$ to $\overrightarrow{\left(e^{1}, e^{1}-\delta e^{1}\right)}$ if some agent has only one neighbor. 


\section{Discussion of the assumptions}

In this section, we discuss various assumptions underlying the main results such as the number of selected agents, the absence of inertia and single active group which means that in each period, only one group of $n$ agents is selected to play the game $\Gamma{ }^{9}$

\subsection{The number of selected agents}

Throughout the analysis, we have maintained the assumption that in each period, a fixed number of agents are randomly chosen to play the minimum effort game $\Gamma$. An interesting question is what happens if the number of selected agents is not constant, but is given by a random variable $\chi$ where $\mathrm{P}\left(\chi=n^{\prime}\right)>0$ for any $n^{\prime} \in\{2,3, \cdots, n\}$ and $\mathrm{P}(\chi=2)+\cdots+\mathrm{P}(\chi=n)=1$.

For simplicity and clarity, assume that the observation network $g$ is connected. In each period, with probability $\mathrm{P}\left(\chi=n^{\prime}\right), n^{\prime} \in\{2,3, \cdots, n\}$ agents are selected to interact. When $\chi=2$ agents are selected, they can exert the highest effort level $e^{m}$ and receive the maximal possible payoff $(1-\delta) e^{m}$. Effort level $e^{m}$ can then spread from these two agents to the entire population. Now consider the exit from $D\left(\left\{\overrightarrow{\left(e^{m}, e^{m}-\delta e^{m}\right)}\right\}\right)$. When agent $i_{0} \in \arg \min _{i \in \mathbf{I}}|M(i)|$ and $n^{\prime}-1$ neighbors (i.e., $\chi=n^{\prime}$ ) are selected to interact, one single mutation by $i_{0}$ can make each of them gets a payoff less than $e^{1}-\delta e^{1}$. To make sure that none of agent $i_{0}$ 's neighbors earns a payoff higher than $e^{1}-\delta e^{1}$ (which would make $i_{0}$ switch back immediately), it is required that other neighbors of $i_{0}$ are consecutively and randomly chosen to play the game $\Gamma$ where each time one agent chooses $e^{1}$ by mistake. The maximal size of the group (i.e., $\chi=n$ ) can lead to the least number of interactions. Formally, for any $e, e^{\prime} \in E$ and $e<e^{\prime}$,

- to induce a transition from $\overrightarrow{(e, e-\delta e)}$ to $\overrightarrow{\left(e^{\prime}, e^{\prime}-\delta e^{\prime}\right)}$, two mutations are both necessary and sufficient; and

- to induce a move from $\overrightarrow{\left(e^{\prime}, e^{\prime}-\delta e^{\prime}\right)}$ to $\overrightarrow{(e, e-\delta e)}, \min _{i \in \mathbf{I}}\left\lceil\frac{|M(i)|}{n}\right\rceil$ mutations are both required and sufficient.

Therefore, the main results are robust to varying interaction group size. Compared to the case where exactly $n$ agents are selected to interact in each period, it is more likely that $\overrightarrow{\left(e^{m}, e^{m}-\delta e^{m}\right)}$ will turn out to be a unique stochastically stable equilibrium.

\subsection{Single active group}

An important aspect of our model is that in each period, only one single group of $n$ agents is selected to interact in a minimum effort game. However, in the real world, when rare and random

\footnotetext{
${ }^{9}$ We would like to thank two anonymous referees so much for pointing out these possibilities and encouraging this discussion.
} 
interaction occurs, several active groups may coexist. A natural question is to ask how robust our main results are to this modification.

Assume that in each period, $\kappa \geq 2$ groups $\mathbf{I}_{1}^{\prime}(t), \cdots, \mathbf{I}_{\kappa}^{\prime}(t)$ of agents are randomly chosen where $(1) \mathbf{I}_{k^{\prime}}^{\prime}(t) \in \mathcal{I}^{n}$ for any $1 \leq k^{\prime} \leq \kappa ;(2) \mathbf{I}_{k^{\prime}}^{\prime}(t) \cap \mathbf{I}_{k^{\prime \prime}}^{\prime \prime}(t)=\emptyset$ for any $1 \leq k^{\prime}, k^{\prime \prime} \leq \kappa$ and $k^{\prime} \neq k^{\prime \prime}$; and (3) all members of each group $\mathbf{I}_{k^{\prime}}^{\prime}(t)$ play the game $\Gamma$. All selected agents interact in separate groups. In other words, each selected agent only interacts with $n-1$ other selected agents. When agent $i \in \mathbf{I}_{k^{\prime}}^{\prime}(t)$ makes a mistake, only the payoffs of $n-1$ other agents from $\mathbf{I}_{k^{\prime}}(t)$ may be influenced. As a result, although two or more active groups coexist, to induce a transition from one absorbing set to any other absorbing set, the same number of mutations are required as in the setting of one single active group. The characterization of stochastically stable equilibria cannot be altered.

\subsection{The absence of inertia}

A common feature of learning dynamics in games is inertia. The imitation rule described in Section 2.3 rules out of inertia. ${ }^{10}$ From a technical point of view, it is important to check whether or not the main results are robust to the introduction of inertia.

Assume that in each period, with common probability $(1-p)$, each selected agent $i$ updates his choice. The probability $p, 0<p<1$, is an indicator of inertia. Suppose that the initial state

is $\overrightarrow{\left(e^{m}, e^{m}-\delta e^{m}\right)}$. Given the existence of inertia, one single mutation is sufficient to induce the transition to $\overrightarrow{\left(e^{1}, e^{1}-\delta e^{1}\right)}$. The reasoning is as follows. In period 0 , the selected agent $i_{0}$ switches to effort level $e^{1}$ by mistake. Then, all members of $M\left(i_{0}\right)$ are consecutively and randomly chosen to participate in the game $\Gamma$ where $i_{0}$ is selected and owing to inertia exerts $e^{1}$ in each interaction. Following the unperturbed dynamics, after a number of periods, $\left\{S^{\epsilon}(t)\right\}_{t \in \mathbb{N}}$ can arrive at a state where the most successful choice in $M\left(i_{0}\right)$ is $e^{1}$. Following the same logic as in the proof of Proposition $1, e^{1}$ can spread to all members of the component $\mathbf{c}$ provided that $i_{0} \in \mathbf{c}$. However, to exit from $D\left(\left\{\overrightarrow{\left(e^{1}, e^{1}-\delta e^{1}\right)}\right\}\right), n$ mutations are required. As a result, $\overrightarrow{\left(e^{m}, e^{m}-\delta e^{m}\right)}$ is not stochastically stable. The assumption of no inertia is indispensable for our main results.

\section{Concluding remark}

The present paper has developed a model of global interaction and local observation where the minimum effort game is repeatedly played by a small proportion of randomly chosen agents. Each selected agent observes the effort levels and payoffs of his neighbors and himself in the most recent

\footnotetext{
${ }^{10}$ In each period, only a small proportion of agents are selected to interact. For any selected agent $i$, it may have been a long time since $i$ has participated the game $\Gamma$ in the last time. Then, it is worthwhile for agent $i$ to evaluate different alternatives again and to revise his choice.
} 
interaction. He imitates the most successful alternative; though occasionally he makes mistake. This paper has shown that in the long run all agents will choose the highest effort level and the socially optimal outcome will obtain, provided that each agent's information neighborhood is large.

The above condition requires that each agent has enough information about others' experiences in the most recent interaction. In other words, observation networks with higher cohesion can produce higher payoffs for individual agents. Therefore, it is tempting to conclude that with declining cost of information acquisition, more efficient outcome can be expected.

There are several natural extensions to the research presented here. First, in practice, it is often the case that in each period, whether or not to participate in a collaboration is for agents to decide, rather than being determined by a random sampling mechanism. To take this into account, the minimum effort game should be extended by incorporating outside option. Second, it would be desirable to consider another interesting case where each agent's payoff increases with the average amount of all players' choices and decreases with the deviation of his own choice. For example, one would like to consider the average opinion game of Van Huyck, Battalio, and Beil (1991). Third, it would be interesting to check whether or not the main results are supported by laboratory experimental examination.

\section{A Proofs}

Proof of Proposition 1. Assume that all agents has been selected to play the game $\Gamma$ until the period $t_{0}$. It is sufficient to prove the conclusion for one component $\mathbf{c}=\left(\mathbf{I}^{\prime},\left(g_{i j}\right)_{i, j \in \mathbf{I}^{\prime}}\right)$. According to the specific value of $\left|\mathbf{I}^{\prime}\right|$, the proof is divided into two cases.

Case 1: $\left|\mathbf{I}^{\prime}\right| \leq n$.

In period $t_{0}+1$, elements of the set $\mathbf{I}^{\prime}\left(t_{0}+1\right) \in \mathcal{I}^{n}, \mathbf{I}^{\prime} \subset \mathbf{I}^{\prime}\left(t_{0}+1\right)$, are randomly chosen to play the game $\Gamma$ with probability $\nu\left(\mathbf{I}^{\prime}\left(t_{0}+1\right)\right)>0$. Each agent $i \in \mathbf{I}^{\prime}$ chooses effort level $e_{i}\left(t_{0}+1\right)$ following Equation (1), and

$$
u_{i}\left(t_{0}+1\right)=\min _{j \in \mathbf{I}_{t_{0}+1}^{\prime}} e_{j}\left(t_{0}+1\right)-\delta e_{i}\left(t_{0}+1\right)
$$

For any two agents $i$ and $i^{\prime}$ from $\mathbf{I}^{\prime}$, if $e_{i}\left(t_{0}+1\right) \geq e_{i^{\prime}}\left(t_{0}+1\right)$, then $u_{i}\left(t_{0}+1\right) \leq u_{i^{\prime}}\left(t_{0}+1\right)$.

In period $t_{0}+2$, agents from the set $\mathbf{I}^{\prime}\left(t_{0}+2\right) \in \mathcal{I}^{n}, \mathbf{I}^{\prime} \subset \mathbf{I}^{\prime}\left(t_{0}+2\right)$, are selected to play the game $\Gamma$ with probability $\nu\left(\mathbf{I}^{\prime}\left(t_{0}+2\right)\right)>0$. According to the deterministic rule, each agent $i \in \mathbf{I}^{\prime}$ chooses $\min _{j \in M(i)} e_{j}\left(t_{0}+1\right)$. The strategy revision follows from the fact that in $M(i)$, the agent choosing the lowest effort level has the best performance in period $t_{0}+1$.

Following the same logic, after a number of periods, $\{S(t)\}_{t \in \mathbb{N}}$ can arrive at a state where all agents from $\mathbf{I}^{\prime}$ exert $\min _{j \in \mathbf{I}^{\prime}} e_{j}\left(t_{0}+1\right)$. 
Case 2: $\left|\mathbf{I}^{\prime}\right|>n$.

Without loss of generality, assume that $\left|\mathbf{I}^{\prime}\right| \leq(2 n-1)$ holds.

For any agent $i \in \mathbf{I}^{\prime}$, let $e_{i}^{\prime}\left(t_{0}+1\right)$ be determined by

$$
e_{i}^{\prime}\left(t_{0}+1\right)=\min \left\{e_{j}\left(t_{0}\right): j \in M(i) \text { and } u_{j}\left(t_{0}\right) \geq u_{j^{\prime}}\left(t_{0}\right) \text { for } \forall j^{\prime} \in M(i)\right\} .
$$

When selected in period $t_{0}+1$, agent $i$ will choose effort level $e_{i}^{\prime}\left(t_{0}+1\right)$ with strictly positive probability. And, $i_{0} \doteq \arg \min _{i \in \mathbf{I}^{\prime}} e_{i}^{\prime}\left(t_{0}+1\right)$.

In period $t_{0}+1$, elements of the set $\mathbf{I}^{\prime}\left(t_{0}+1\right) \in \mathcal{I}^{n}$ are randomly chosen to play the game $\Gamma$ with probability $\nu\left(\mathbf{I}^{\prime}\left(t_{0}+1\right)\right)>0$ where $i_{0} \in \mathbf{I}^{\prime}\left(t_{0}+1\right)$ and $\mathbf{I}^{\prime}\left(t_{0}+1\right) \subset \mathbf{I}^{\prime}$. Suppose that each agent $i \in \mathbf{I}^{\prime}\left(t_{0}+1\right)$ chooses $e_{i}^{\prime}\left(t_{0}+1\right)$. Thus, for any $i \in \mathbf{I}^{\prime}\left(t_{0}+1\right)$,

$$
\begin{aligned}
u_{i}\left(t_{0}+1\right) & =\min _{j \in \mathbf{I}_{t_{0}+1}^{\prime}} e_{j}\left(t_{0}+1\right)-\delta e_{i}\left(t_{0}+1\right)=e_{i_{0}}^{\prime}\left(t_{0}+1\right)-\delta e_{i}^{\prime}\left(t_{0}+1\right) \\
& \leq(1-\delta) e_{i_{0}}^{\prime}\left(t_{0}+1\right) .
\end{aligned}
$$

In particular, $u_{i_{0}}\left(t_{0}+1\right)=(1-\delta) \times e_{i_{0}}^{\prime}\left(t_{0}+1\right)$.

In period $t_{0}+2$, agents belonging to the set $\mathbf{I}^{\prime}\left(t_{0}+2\right) \in \mathcal{I}^{n}$ are selected to play the game $\Gamma$ with probability $\nu\left(\mathbf{I}^{\prime}\left(t_{0}+2\right)\right)>0$ where $\left\{i_{0}\right\} \cup\left(\mathbf{I}^{\prime} \backslash \mathbf{I}^{\prime}\left(t_{0}+1\right)\right) \subset \mathbf{I}^{\prime}\left(t_{0}+2\right)$. On the one hand, agent $i_{0}$ chooses $e_{i_{0}}^{\prime}\left(t_{0}+1\right)$. For any agent $j \in M\left(i_{0}\right),(1)$ if $j \in \mathbf{I}^{\prime}\left(t_{0}+1\right), u_{j}\left(t_{0}+1\right) \leq u_{i_{0}}\left(t_{0}+1\right)$ and $(2)$ if $j \notin \mathbf{I}^{\prime}\left(t_{0}+1\right), u_{j}\left(t_{0}+1\right)=u_{j}\left(t_{0}\right) \leq \max _{j^{\prime} \in M\left(i_{0}\right)} u_{j^{\prime}}\left(t_{0}\right) \leq(1-\delta) e_{i_{0}}^{\prime}\left(t_{0}+1\right)=u_{i_{0}}\left(t_{0}+1\right)$ where the second inequality follows from the determination of $e_{i_{0}}^{\prime}\left(t_{0}+1\right)$ and the fact that the maximal payoff brought about by $e_{i_{0}}^{\prime}\left(t_{0}+1\right)$ is $(1-\delta) \times e_{i_{0}}^{\prime}\left(t_{0}+1\right)$. On the other hand, for any $i \in \mathbf{I}^{\prime}\left(t_{0}+2\right) \backslash\left\{i_{0}\right\}$, agent $i$ 's choice $e_{i}\left(t_{0}+2\right)$ satisfies that

$$
\begin{aligned}
e_{i}\left(t_{0}+2\right) & \in\left\{e_{k}\left(t_{0}+1\right): k \in M(i)\right\} \\
& \subseteq \cup_{k \in M(i)}\left\{e_{j}\left(t_{0}\right): j \in M(k) \text { and } u_{j}\left(t_{0}\right) \geq u_{j^{\prime}}\left(t_{0}\right) \text { for } \forall j^{\prime} \in M(k)\right\} .
\end{aligned}
$$

Due to the determination of $e_{i_{0}}^{\prime}\left(t_{0}+1\right), e_{i}\left(t_{0}+2\right) \geq e_{i_{0}}^{\prime}\left(t_{0}+1\right)=e_{i_{0}}\left(t_{0}+2\right)$. Thus, $\{S(t)\}_{t \in \mathbb{N}}$ can arrive at a state $s\left(t_{0}+2\right)$ where for any $i \in \mathbf{I}^{\prime}$, agent $i$ 's state is $\left(e_{i}\left(t_{0}+2\right), e_{i_{0}}^{\prime}\left(t_{0}+1\right)-\delta e_{i}\left(t_{0}+2\right)\right)$ with $e_{i}\left(t_{0}+2\right) \geq e_{i_{0}}^{\prime}\left(t_{0}+1\right)$.

In period $t_{0}+3$, agents belonging to the set $\mathbf{I}^{\prime}\left(t_{0}+3\right) \in \mathcal{I}^{n}$ are randomly chosen to play the game $\Gamma$ with probability $\nu\left(\mathbf{I}^{\prime}\left(t_{0}+3\right)\right)>0$ where $\mathbf{I}^{\prime}\left(t_{0}+3\right) \subset M\left(i_{0}\right)$ if $\left|M\left(i_{0}\right)\right| \geq n$; otherwise, $M\left(i_{0}\right) \subset \mathbf{I}^{\prime}\left(t_{0}+3\right)$. According to the deterministic imitation rule, each agent $i \in \mathbf{I}^{\prime}\left(t_{0}+3\right) \cap M\left(i_{0}\right)$ chooses $e_{i_{0}}^{\prime}\left(t_{0}+1\right)$. The strategy revision owes to the fact that for any $i \in \mathbf{I}^{\prime}, u_{i}\left(t_{0}+2\right) \leq$ $(1-\delta) e_{i_{0}}^{\prime}\left(t_{0}+1\right)=u_{i_{0}}\left(t_{0}+2\right)$ and the equality holds only if $e_{i}\left(t_{0}+2\right)=e_{i_{0}}^{\prime}\left(t_{0}+1\right)$.

Following the same logic, after a number of periods, $\{S(t)\}_{t \in \mathbb{N}}$ can reach a state where all agents from $\mathbf{I}^{\prime}$ choose $e_{i_{0}}^{\prime}\left(t_{0}+1\right)$.

Proof of Lemma 1. It is sufficient to consider the case where $\eta(g)=2$. Formally, the interaction system $(\mathbf{I}, g)$ consists of $\mathbf{c}_{1}=\left(\mathbf{I}_{1}^{\prime},\left(g_{i j}\right)_{i, j \in \mathbf{I}_{1}^{\prime}}\right)$ and $\mathbf{c}_{2}=\left(\mathbf{I}_{2}^{\prime},\left(g_{i j}\right)_{i, j \in \mathbf{I}_{2}^{\prime}}\right)$. Suppose that in 
some period $t_{0},\left\{S^{\epsilon}(t)\right\}_{t \in \mathbb{N}}$ arrives at the state $s^{\epsilon}\left(t_{0}\right)$ where $s_{i}^{\epsilon}\left(t_{0}\right)=\left(e^{l}(\bar{\Omega}),(1-\delta) e^{l}(\bar{\Omega})\right)$ for all $i \in \mathbf{I}_{1}^{\prime}$ and $s_{j}^{\epsilon}\left(t_{0}\right)=\left(e^{h}(\bar{\Omega}), e^{l}(\bar{\Omega})-\delta e^{h}(\bar{\Omega})\right)$ for all $j \in \mathbf{I}_{2}^{\prime}$. That is, each agent $j \in \mathbf{I}_{2}^{\prime}$ has played the game $\Gamma$ with some agent from $\mathbf{I}_{1}^{\prime}$ in the most recent interaction.

In period $t_{0}+1$, agents from the set $\mathbf{I}^{\prime}\left(t_{0}+1\right) \in \mathcal{I}^{n}$ are selected to play the game $\Gamma$ with probability $\nu\left(\mathbf{I}^{\prime}\left(t_{0}+1\right)\right)>0$ where $\mathbf{I}^{\prime}\left(t_{0}+1\right) \cap \mathbf{I}_{1}^{\prime} \neq \emptyset$ and $\mathbf{I}^{\prime}\left(t_{0}+1\right) \cap \mathbf{I}_{2}^{\prime} \neq \emptyset$. Owing to the deterministic rule, each agent $i \in \mathbf{I}^{\prime}\left(t_{0}+1\right) \cap \mathbf{I}_{1}^{\prime}$ should exert $e^{l}(\bar{\Omega})$ and each agent $j \in \mathbf{I}^{\prime}\left(t_{0}+1\right) \cap \mathbf{I}_{2}^{\prime}$ should choose $e^{h}(\bar{\Omega})$. However, agent $j_{0} \in \mathbf{I}^{\prime}\left(t_{0}+1\right) \cap \mathbf{I}_{2}^{\prime}$ makes a mistake and chooses effort level $e^{l}(\bar{\Omega})$. Then, $s_{j_{0}}^{\epsilon}\left(t_{0}+1\right)=\left(e^{l}(\bar{\Omega}),(1-\delta) e^{l}(\bar{\Omega})\right)$ and $s_{j}^{\epsilon}\left(t_{0}+1\right)=\left(e^{h}(\bar{\Omega}), e^{l}(\bar{\Omega})-\delta e^{h}(\bar{\Omega})\right)$ for any $j \in \mathbf{I}_{2}^{\prime} \backslash\left\{j_{0}\right\}$. According to the unperturbed imitation rule, members of $M\left(j_{0}\right)$ will choose $e^{l}(\bar{\Omega})$ if selected in period $t_{0}+2$.

Following the same logic as in the proof of Proposition 1, after a number of periods, $\left\{S^{\epsilon}(t)\right\}_{t \in \mathbb{N}}$ can arrive at $\overrightarrow{\left(e^{l}(\bar{\Omega}), e^{l}(\bar{\Omega})-\delta e^{l}(\bar{\Omega})\right)}$.

In each period $t, t=0,1, \ldots$, let $e^{h}(s(t))$ denote the highest effort level chosen by agents in the state $s(t)$; formally, $e_{i}(t) \leq e^{h}(s(t))$ for any $i \in \mathbf{I}$ and $e_{i_{0}}(t)=e^{h}(s(t))$ for some agent $i_{0} \in \mathbf{I}$. Owing to the deterministic imitation rule, $e^{h}(s(t))$ cannot strictly increase as time moves on. Let $\hat{\mathbf{I}}(t) \doteq\left\{i \in \mathbf{I}: s_{i}(t)=\left(e^{h}(s(t)),(1-\delta) e^{h}(s(t))\right)\right\}$ denote the set of agents who have chosen $e^{h}(s(t))$ and received the maximal payoff $(1-\delta) e^{h}(s(t))$ brought about by $e^{h}(s(t))$ in the most recent interaction until period $t+1$. The following lemma is essential for the proof of Lemma 2 .

Lemma 4. Consider the unperturbed dynamics $\{S(t)\}_{t \in \mathbb{N}}$. Assume that in some period $t_{0},\left|\hat{\mathbf{I}}\left(t_{0}\right)\right| \geq$ $n$ holds. Then, $\{S(t)\}_{t \in \mathbb{N}}$ can converge to an absorbing set where for any component $\mathbf{c}=$ $\left(\mathbf{I}^{\prime},\left(g_{i j}\right)_{i, j \in \mathbf{I}^{\prime}}\right)$, all members exert effort level $e^{h}\left(s\left(t_{0}\right)\right)$ if $\mathbf{I}^{\prime} \cap \hat{\mathbf{I}}\left(t_{0}\right) \neq \emptyset$.

Proof. It is sufficient to prove the conclusion for one component $\mathbf{c}=\left(\mathbf{I}^{\prime},\left(g_{i j}\right)_{i, j \in \mathbf{I}^{\prime}}\right)$ where $\mathbf{I}^{\prime} \cap \hat{\mathbf{I}}\left(t_{0}\right) \neq \emptyset$ and $\mathbf{I}^{\prime} \backslash \hat{\mathbf{I}}\left(t_{0}\right) \neq \emptyset$.

In period $t_{0}+1$, agents belonging to the set $\mathbf{I}^{\prime}\left(t_{0}+1\right) \in \mathcal{I}^{n}$ are selected to play the game $\Gamma$ with probability $\nu\left(\mathbf{I}^{\prime}\left(t_{0}+1\right)\right)>0$ where both $\left(\mathbf{I}^{\prime}\left(t_{0}+1\right) \cap \mathbf{I}^{\prime}\right) \backslash \hat{\mathbf{I}}\left(t_{0}\right) \neq \emptyset$ and $\mathbf{I}^{\prime}\left(t_{0}+1\right) \subset \cup_{j \in \hat{\mathbf{I}}\left(t_{0}\right)} M(j)$ hold. The existence of $\mathbf{I}^{\prime}\left(t_{0}+1\right)$ follows from the fact that $\left|\hat{\mathbf{I}}\left(t_{0}\right)\right| \geq n, \mathbf{I}^{\prime} \cap \hat{\mathbf{I}}\left(t_{0}\right) \neq \emptyset$ and $\mathbf{I}^{\prime} \backslash \hat{\mathbf{I}}\left(t_{0}\right) \neq$ $\emptyset$. According to the deterministic rule given by Equation (1), all members of $\mathbf{I}^{\prime}\left(t_{0}+1\right)$ choose effort level $e^{h}\left(s\left(t_{0}\right)\right)$. The strategy revisions result from the fact that $\mathbf{I}^{\prime}\left(t_{0}+1\right) \subset \cup_{j \in \hat{\mathbf{I}}\left(t_{0}\right)} M(j)$. Thus, for any $i \in \mathbf{I}^{\prime}\left(t_{0}+1\right)$,

$$
e_{i}\left(t_{0}+1\right)=e^{h}\left(s\left(t_{0}\right)\right) \text { and } u_{i}\left(t_{0}+1\right)=(1-\delta) e^{h}\left(s\left(t_{0}\right)\right) .
$$

It follows that $\hat{\mathbf{I}}\left(t_{0}+1\right)=\hat{\mathbf{I}}\left(t_{0}\right) \cup \mathbf{I}^{\prime}\left(t_{0}+1\right)$ and $\hat{\mathbf{I}}\left(t_{0}\right) \subsetneq \hat{\mathbf{I}}\left(t_{0}+1\right)$.

Following the same logic, after a number of periods, $\{S(t)\}_{t \in \mathbb{N}}$ can arrive at a state where all members of $\mathbf{c}$ choose $e^{h}\left(s\left(t_{0}\right)\right)$. 
Proof of Lemma 2. Without loss of generality, assume that the interaction system $(\mathbf{I}, g)$ consists of $\eta(g)=n+1$ components. Suppose that in some period $t_{0},\left\{S^{\epsilon}(t)\right\}_{t \in \mathbb{N}}$ arrives at $\overrightarrow{(e, e-\delta e)}$.

Part 1: Transition from $\overrightarrow{(e, e-\delta e)}$ to $\bar{\Omega}_{1}$.

In period $t_{0}+1$, members of the group $\mathbf{I}^{\prime}\left(t_{0}+1\right) \in \mathcal{I}^{n}$ are randomly chosen to play the game $\Gamma$ with probability $\nu\left(\mathbf{I}^{\prime}\left(t_{0}+1\right)\right)>0$ where any two distinct selected agents are from different components. By mistake, each agent $i \in \mathbf{I}^{\prime}\left(t_{0}+1\right)$ chooses effort level $e^{\prime}$ rather than $e$. Agent $i$ then receives a payoff $(1-\delta) e^{\prime}$. Owing to the definition of the game $\Gamma, n$ mistakes are sufficient and necessary.

$\mathbf{I}^{\prime}\left(t_{0}+1\right)$ can play the role of the set $\hat{\mathbf{I}}\left(t_{0}\right)$ in Lemma 4. Following the unperturbed dynamics, in some period $t_{1},\left\{S^{\epsilon}(t)\right\}_{t \in \mathbb{N}}$ can arrive at the absorbing set $\bar{\Omega}_{1}$ where there is a component $\mathbf{c}=\left(\mathbf{I}^{\prime},\left(g_{i j}\right)_{i, j \in \mathbf{I}^{\prime}}\right)$ such that all members of $\mathbf{c}$ exert $e$ while all elements of $\mathbf{I} \backslash \mathbf{I}^{\prime}$ choose $e^{\prime}$. And, both $e^{h}\left(\bar{\Omega}_{1}\right)=e^{\prime}$ and $\rho\left(\bar{\Omega}_{1}\right) \geq n$ hold.

Part 2: Transition from $\bar{\Omega}_{1}$ to $\overrightarrow{\left(e^{\prime}, e^{\prime}-\delta e^{\prime}\right)}$.

In period $t_{1}+1$, a group $\mathbf{I}^{\prime}\left(t_{1}+1\right)$ of $n$ agents is selected to play the game $\Gamma$ with probability $\nu\left(\mathbf{I}^{\prime}\left(t_{1}+1\right)\right)>0$ where $\mathbf{I}^{\prime}\left(t_{1}+1\right) \cap \mathbf{I}^{\prime}=\left\{i_{0}\right\}$ is a singleton. According to the deterministic imitation rule, each agent $j \in \mathbf{I}^{\prime}\left(t_{1}+1\right) \backslash\left\{i_{0}\right\}$ should choose $e^{\prime}$ while agent $i_{0}$ should choose $e$. Due to one single mutation, all agents from $\mathbf{I}^{\prime}\left(t_{1}+1\right)$ exert $e^{\prime}$. Thus, for any agent $i \in \mathbf{I}^{\prime}\left(t_{1}+1\right),{ }^{11}$

$$
e_{i}\left(t_{1}+1\right)=e^{\prime} \text { and } u_{i}\left(t_{1}+1\right)=(1-\delta) e^{\prime}>(1-\delta) e .
$$

$\left(\mathbf{I} \backslash \mathbf{I}^{\prime}\right) \cup\left\{i_{0}\right\}$ can play the role of $\hat{\mathbf{I}}\left(t_{0}\right)$ in Lemma 4. Following the unperturbed dynamics, $\left\{S^{\epsilon}(t)\right\}_{t \in \mathbb{N}}$ can converge to $\overline{\left(e^{\prime}, e^{\prime}-\delta e^{\prime}\right)}$.

It is also likely that all members of a component $\mathbf{c}^{\prime}$ are selected in one single period and each of them makes a mistake when $\left|\mathbf{c}^{\prime}\right| \leq n$. In combination with the above proof, $\min \left\{n, \min _{\mathbf{c} \subseteq \mathbf{g}}|\mathbf{c}|\right\}$ mutations are required and sufficient to induce an exit from $D\left(\left\{\overrightarrow{\left(e^{1}, e^{1}-\delta e^{1}\right)}\right\}\right)$.

Proof of Lemma 3. Consider agent $i_{0} \in \arg \min _{i \in \mathbf{I}}|M(i)|$. Without loss of generality, assume that $n<\left|M\left(i_{0}\right)\right| \leq 2 n$. Suppose that in some period $t_{0},\left\{S^{\epsilon}(t)\right\}_{t \in \mathbb{N}}$ arrives at $\left(e^{\prime} \mathbf{1},(1-\delta) e^{\prime} \mathbf{1}\right)$.

In period $t_{0}+1$, agents belonging to the set $\mathbf{I}^{\prime}\left(t_{0}+1\right) \in \mathcal{I}^{n}$ are selected to play the game $\Gamma$ with probability $\nu\left(\mathbf{I}^{\prime}\left(t_{0}+1\right)\right)>0$ where $i_{0} \in \mathbf{I}^{\prime}\left(t_{0}+1\right)$ and $\mathbf{I}^{\prime}\left(t_{0}+1\right) \subset M\left(i_{0}\right)$. The deterministic imitation rule stipulates the choice of $e^{\prime}$ for any agent $i \in \mathbf{I}^{\prime}\left(t_{0}+1\right)$. Each agent $j \in \mathbf{I}^{\prime}\left(t_{0}+1\right) \backslash\left\{i_{0}\right\}$ chooses $e^{\prime}$. And, by mistake, agent $i_{0}$ chooses effort level $e$. As a result,

$$
e_{i_{0}}\left(t_{0}+1\right)=e, u_{i_{0}}\left(t_{0}+1\right)=(1-\delta) e
$$

\footnotetext{
${ }^{11}$ For simplicity and clarity, we ignore the dependence of each agent's state on the probability $\epsilon$.
} 
and for any $j \in \mathbf{I}^{\prime}\left(t_{0}+1\right) \backslash\left\{i_{0}\right\}$,

$$
e_{j}\left(t_{0}+1\right)=e^{\prime}, u_{j}\left(t_{0}+1\right)=e-\delta e^{\prime}<(1-\delta) e=u_{i_{0}}\left(t_{0}+1\right) \text {. }
$$

In period $t_{0}+2$, members of the group $\mathbf{I}^{\prime}\left(t_{0}+2\right) \in \mathcal{I}^{n}$ are selected to play game $\Gamma$ with probability $\nu\left(\mathbf{I}^{\prime}\left(t_{0}+2\right)\right)>0$ where $M\left(i_{0}\right) \backslash \mathbf{I}^{\prime}\left(t_{0}+1\right) \subset \mathbf{I}^{\prime}\left(t_{0}+2\right)$. The existence of $\mathbf{I}^{\prime}\left(t_{0}+2\right)$ follows from the fact that $n<\left|M\left(i_{0}\right)\right| \leq 2 n$. Owing to the deterministic imitation rule, each agent $j \in M\left(i_{0}\right) \backslash \mathbf{I}^{\prime}\left(t_{0}+1\right)$ should choose $e^{\prime}$. In fact, for any $j \in M\left(i_{0}\right) \backslash \mathbf{I}^{\prime}\left(t_{0}+1\right)$, (1) $M(j) \backslash \mathbf{I}^{\prime}\left(t_{0}+1\right) \neq \emptyset$; otherwise, $|M(j)| \leq n<\left|M\left(i_{0}\right)\right|$ which contradicts the determination of $i_{0}$ and $(2)$ for any $k \in M(j) \backslash \mathbf{I}^{\prime}\left(t_{0}+1\right), s_{k}\left(t_{0}+1\right)=\left(e^{\prime},(1-\delta) e^{\prime}\right)$. Due to one single mutation, one agent $j_{0} \in \mathbf{I}^{\prime}\left(t_{0}+2\right)$ exerts $e$ while each agent $i \in \mathbf{I}^{\prime}\left(t_{0}+2\right) \backslash\left\{j_{0}\right\}$ chooses $e^{\prime}$.

Therefore, after $\min _{i \in \mathbf{I}}\left\lceil\frac{|M(i)|}{n}\right\rceil(=2)$ mutations, $\left\{S^{\epsilon}(t)\right\}_{t \in \mathbb{N}}$ can arrive at a state where

$$
e_{i_{0}}\left(t_{0}+2\right)=e_{j_{0}}\left(t_{0}+2\right)=e, u_{i_{0}}\left(t_{0}+2\right)=u_{j_{0}}\left(t_{0}+2\right)=(1-\delta) e
$$

and for any $j \in M\left(i_{0}\right) \backslash\left\{i_{0}, j_{0}\right\}$,

$$
e_{j}\left(t_{0}+2\right)=e^{\prime}, u_{j}\left(t_{0}+2\right)=e-\delta e^{\prime}<(1-\delta) e=u_{i_{0}}\left(t_{0}+2\right) \text {. }
$$

According to the deterministic imitation rule, agent $i_{0}$ will choose $e$ if selected in the next period.

Following the same logic as in the proof of Proposition 1, without any further mutation, $\left\{S^{\epsilon}(t)\right\}_{t \in \mathbb{N}}$ can converge to the absorbing set $\bar{\Omega}_{1}$ where all members of the component c exert $e$ provided that $i_{0} \in \mathbf{c}$. The transition from $\bar{\Omega}_{0}$ to $\bar{\Omega}_{1}$ is completed. The inference shows that $\min _{i \in \mathbf{I}}\left\lceil\frac{|M(i)|}{n}\right\rceil$ mutations are necessary.

For the absorbing set $\bar{\Omega}_{1}$, it follows that

$$
e^{h}\left(\bar{\Omega}_{1}\right)=e^{\prime}>e=e^{l}\left(\bar{\Omega}_{1}\right) .
$$

The remaining part of the proof is simply an application of Lemma 1.

The above proof also implies that to induce an exit from $\left.D\left(\left\{\overrightarrow{\left(e^{m}, e^{m}-\delta e^{m}\right.}\right)\right\}\right), \min _{i \in \mathbf{I}}\left\lceil\frac{|M(i)|}{n}\right\rceil$ mutations are both necessary and sufficient.

Proof of Theorem 1. The proof uses the modified radius-coradius theorem developed by Ellison (2000). According to the relationship between $\min _{i \in \mathbf{I}}\left\lceil\frac{|M(i, g)|}{n}\right\rceil$ and $n$, the proof is divided into three cases. We restrict our attention to the case where $\min _{i \in \mathbf{I}}\left\lceil\frac{|M(i, g)|}{n}\right\rceil>n$. The other two cases can be proved in a similar fashion. When $\min _{i \in \mathbf{I}}\left\lceil\frac{|M(i, g)|}{n}\right\rceil=n$, it is supposed to explore the radius and modified-coradius of the set $\{\overrightarrow{(e, e-\delta e)}: e \in E\}$. 
First of all, by Lemma 3, we have that ${ }^{12}$

$$
\left.R\left(\left\{\overrightarrow{\left(e^{m}, e^{m}-\delta e^{m}\right.}\right)\right\}\right)=\min _{i \in \mathbf{I}}\left\lceil\frac{|M(i, g)|}{n}\right\rceil .
$$

To calculate $C R^{*}\left(\left\{\overrightarrow{\left(e^{m}, e^{m}-\delta e^{m}\right)}\right\}\right)$, it is sufficient to consider the transition from $\overrightarrow{\left(e^{1}, e^{1}-\delta e^{1}\right)}$ to $\overrightarrow{\left(e^{m}, e^{m}-\delta e^{m}\right)}$. It follows that

$$
\begin{aligned}
& C R^{*}\left(\left\{\overrightarrow{\left(e^{m}, e^{m}-\delta e^{m}\right)}\right\}\right)={ }_{s \notin D}\left(\left\{\frac{\max }{\left(e^{m}, e^{m}-\delta e^{m}\right)}\right\}\right) r^{*}\left(s, \overrightarrow{\left(e^{m}, e^{m}-\delta e^{m}\right)}\right) \\
& =r^{*}\left(\overrightarrow{\left(e^{1}, e^{1}-\delta e^{1}\right)}, \overrightarrow{\left(e^{m}, e^{m}-\delta e^{m}\right)}\right) \\
& =[n+(\max \{n, \eta(g)\}-n)]-(\max \{n, \eta(g)\}-n) \\
& =n
\end{aligned}
$$

where the third equation owes to the fact that following from the proof of Lemma 2, there exists a sequence of distinct absorbing sets $\left(\bar{\Omega}_{0}, \cdots, \bar{\Omega}_{k}\right), k=\eta(g)+1-\min \{n, \eta(g)\}$, such that (1) $\bar{\Omega}_{0}=\left\{\overrightarrow{\left(e^{1}, e^{1}-\delta e^{1}\right)}\right\}$ and $\bar{\Omega}_{k}=\left\{\overrightarrow{\left(e^{m}, e^{m}-\delta e^{m}\right)}\right\}$; (2) for $\bar{\Omega}_{1}, \min \{n, \eta(g)\}$ components have all of their members choose $e^{m}$ and $\bar{\Omega}_{k^{\prime}+1}$ is such that there is one more component with all members choosing $e^{m}$ than in $\bar{\Omega}_{k^{\prime}}$ when $k^{\prime}=1, \cdots,(k-1) ;(3)$ to transit from $\left\{\overrightarrow{\left(e^{1}, e^{1}-\delta e^{1}\right)}\right\}$ to $\bar{\Omega}_{1}, n$ mistakes are required and sufficient and one single mutation can induce a transition from $\bar{\Omega}_{k^{\prime}}$ to $\bar{\Omega}_{k^{\prime}+1}$ for any $1 \leq k^{\prime} \leq(k-1)$; and $(4) R\left(\bar{\Omega}_{k^{\prime}}\right)=1$ for any $1 \leq k^{\prime} \leq(k-1)$.

Consequently, if $\left.\min _{i \in \mathbf{I}}\left\lceil\frac{|M(i, g)|}{n}\right\rceil>n, R\left(\left\{\overrightarrow{\left(e^{m}, e^{m}-\delta e^{m}\right.}\right)\right\}\right)>C R^{*}\left(\left\{\overrightarrow{\left(e^{m}, e^{m}-\delta e^{m}\right.}\right\}\right)$. As an application of the modified radius-coradius theorem in Ellison (2000), only $\overrightarrow{\left(e^{m}, e^{m}-\delta e^{m}\right)}$ is stochastically stable.

\section{References}

[1] Alós-Ferrer, C., 2004. "Cournot versus Walras in dynamic oligopolies with memory." International Journal of Industrial Organization 22, 193-217.

[2] Alós-Ferrer, C., 2008. "Learning, bounded memory, and inertia." Economics Letters 101, 134-136.

[3] Alós-Ferrer, C., Weidenholzer, S., 2007. "Partial bandwagon effects and local interactions." Games and Economic Behavior 61, 179-197.

[4] Alós-Ferrer, C., Weidenholzer, S., 2008. "Contagion and efficiency." Journal of Economic Theory 143, 251-274.

\footnotetext{
${ }^{12}$ For any union of absorbing sets $\bar{\Omega}, R(\bar{\Omega})$ and $C R^{*}(\bar{\Omega})$ are the radius and modified coradius of the set $\bar{\Omega}$ respectively.
} 
[5] Alós-Ferrer, C., Weidenholzer, S., 2014. "Imitation and the role of information in overcoming coordination failures." Games and Economic Behavior 87, 397-411.

[6] Anderlini, L., Ianni, A., 1996. "Path dependence and learning from neighbors." Games and Economic Behavior 13, 141-177.

[7] Angus, S., Masson, V., 2010. "The effects of information and interactions on contagion processes." Working Paper.

[8] Bergin, J., Bernhardt, D., 2009. “Cooperation through imitation.” Games and Economic Behavior 67, 376-388.

[9] Blume, L.E., 1993. “The statistical mechanics of strategic interaction.” Games and Economic Behavior 5, 387-424.

[10] Blume, L.E., 1995. “The statistical mechanics of best-response strategy revision.” Games and Economic Behavior 11, 111-145.

[11] Boyer, T., Jonard, N., 2014. "Imitation and efficient contagion." Journal of Economic Behavior \& Organization 100, 20-32.

[12] Cui, Z., 2014. "More neighbors, more efficiency.” Journal of Economic Dynamics \& Control 40, 103-115.

[13] Ellison, G., 1993. "Learning, local interaction, and coordination.” Econometrica 61, 10471071.

[14] Ellison, G., 2000. "Basins of attraction, long-run stochastic stability, and the speed of stepby-step evolution." Review of Economic Studies 67, 17-45.

[15] Eshel, I., Samuelson, L., Shaked, A., 1998. "Altruists, egoists, and hooligans in a local interaction model." American Economic Review 88, 157-179.

[16] Goyal, S., 2011. "Learning in networks.” In: Benhabib, J., Bisin, A., Jackson, M., (Eds.) Handbook of Social Economics, Volume 1, North Holland.

[17] Khan, A., 2014. "Coordination under global random interaction and local imitation." International Journal of Game Theory 43, 721-745.

[18] Kandori, M., Mailath, G., Rob, R., 1993. "Learning, mutation, and long run equilibria in games." Econometrica 61, 29-56.

[19] Özgür, O., 2011. “Local interactions.” In: Benhabib, J., Bisin, A., Jackson, M., (Eds.) Handbook of Social Economics, Volume 1, North Holland. 
[20] Morris, S., 2000. “Contagion.” Review of Economic Studies 67, 57-78.

[21] Robson, A.J., Vega-Redondo, S., 1996. "Efficient equilibrium selection in evolutionary games with random matching." Journal of Economic Theory 70, 65-92.

[22] Seidman, S.B., 1983. "Network structure and minimum degree." Social Networks 5, 269287.

[23] Van Huyck, J., Battalio, R., Beil, R., 1990. "Tacit coordination games, strategic uncertainty, and coordination failure.” American Economic Review 80, 234-248.

[24] Van Huyck, J., Battalio, R., Beil, R., 1991. "Strategic uncertainty, equilibrium selection, and coordination failure in average opinion games." Quarterly Journal of Economics 106, 234248.

[25] Weber, R., 2006. "Managing growth to achieve efficient coordination in large groups." American Economic Review 96, 114-126.

[26] Weidenholzer, S., 2010. "Coordination games and local interactions: A survey of the game theoretic literature." Games 1, 885-910.

[27] Young, H.P., 1993. “The evolution of conventions.” Econometrica 61, 57-84. 

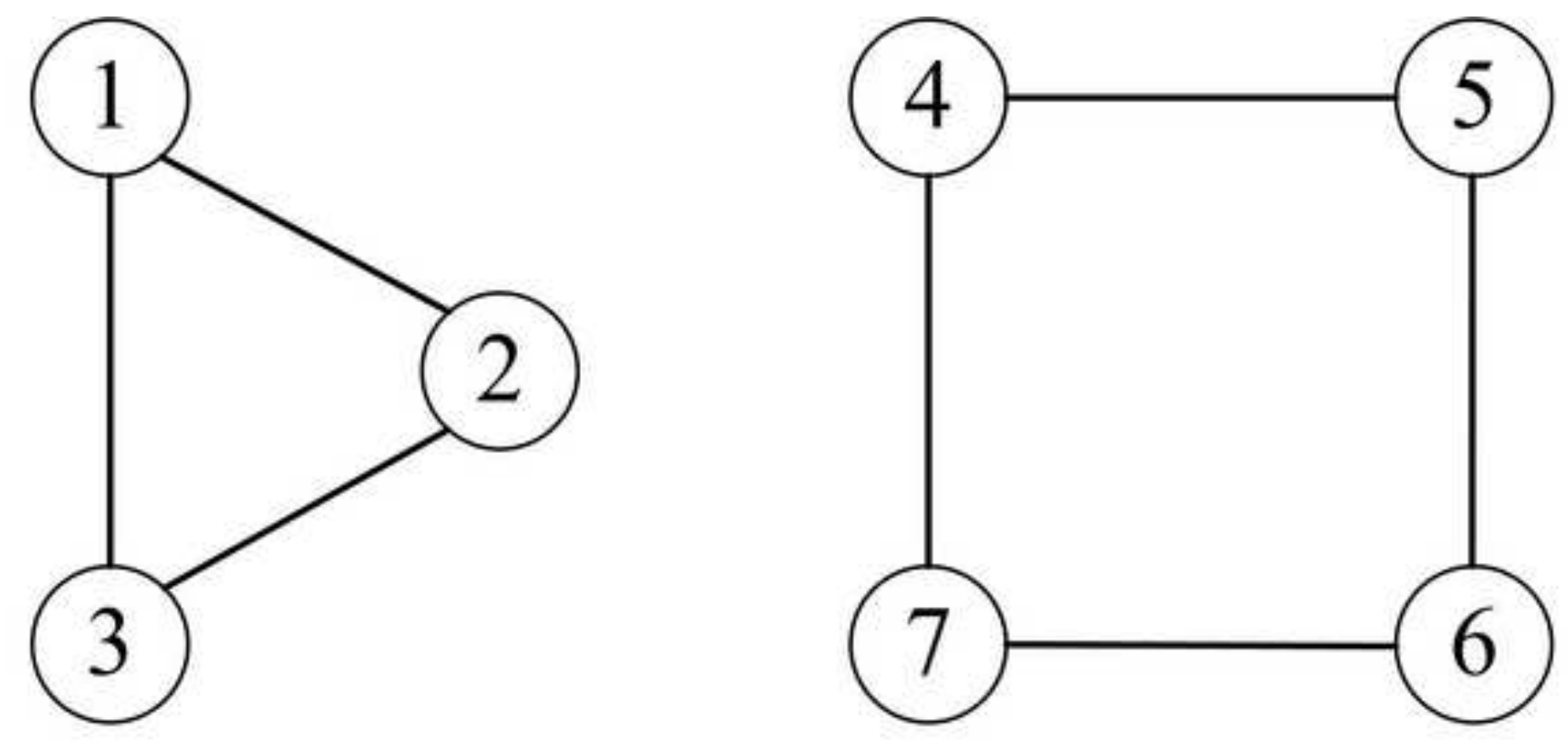

Figure 1: An observation system consisting of two components. 